\title{
ALMOST EVERYWHERE CONVERGENCE FOR SEQUENCES OF CONTINUOUS FUNCTIONS
}

\author{
K. SCHR ADER AND S. UMAMAHESWARAM
}

ABSTRACT. The main result in this paper is the following theorem.

Theorem 1.1. Let $\left\{y_{k}\right\}, y_{k}: I \rightarrow R$, be a sequence of continuous real valued functions defined on the bounded interval $I$. Let $D_{k j}=$ $\left\{x: x \in I,\left|y_{k}(x)-y_{j}(x)\right|>0\right\}=\bigcup_{n} I_{k j n}$, where each $I_{k j n}$ is a relatively open subinterval of $I$ and $I_{k j n} \cap I_{k j m}=\varnothing$ for $n \neq m$. Assume there exists a function $\phi, \phi:(0,+\infty) \rightarrow(0,+\infty)$, such that $\lim _{r \rightarrow 0^{+}}(\phi(r) / r)=$ $+\infty, \phi$ is a nondecreasing function of $r$ and

$$
\sum_{n} \phi\left(\mu\left(I_{k j n}\right)\right) \sup _{x \in I_{k j n}}\left|y_{k}(x)-y_{j}(x)\right|^{p} \leq M
$$

for all $k, j$ sufficiently large, where $\mu$ is Lebesgue measure and $0<$ $p<+\infty$. Then $\left\{y_{k}\right\}$ contains a subsequence which converges almost everywhere to a Lebesgue measurable function $y$.

1. Introduction. Let $R$ be the real numbers, $I \subset R$ be a bounded interval and $\left\{y_{k}\right\}, y_{k}: I \rightarrow R$, be a sequence of continuous functions. In this paper we give a sufficient condition that $\left\{y_{k}\right\}$ have a subsequence which converges almost everywhere with respect to Lebesgue measure $\mu$ on the interval $I$. In particular, we establish the following theorem.

Theorem 1.1. Let $\left\{y_{k}\right\}, y_{k}: I \rightarrow R$ be a sequence of continuous real valued functions defined on the bounded interval I. Let $D_{k j}=\{x: x \in I$, $\left.\left|y_{k}(x)-y_{j}(x)\right|>0\right\}=\bigcup_{n} I_{k j n}$, where each $I_{k j n}$ is a relatively open subinterval of $I$ and $I_{k j n} \cap I_{k j m}=\varnothing$ for $n \neq m$. Assume there exists a function $\phi, \phi$ : $(0,+\infty) \rightarrow(0,+\infty)$, such that $\lim _{r-\rightarrow 0^{+}}(\phi(r) / r)=+\infty, \phi$ is a nondecreasing function of $r$ and

$$
\sum_{n} \phi\left(\mu\left(I_{k j n}\right)\right) \sup _{x \in I_{k j n}}\left|y_{k}(x)-y_{j}(x)\right|^{p} \leq M
$$

Received by the editors January 23, 1974.

AMS (MOS) subject classifications (1970). Primary $40 \mathrm{~A} 05$.

Key words and phrases. Sequences of functions, convergent subsequences, almost everywhere convergence. 
for all $k, j$ sufficiently large, where $\mu$ is Lebesgue measure and $0<p<+\infty$. Then $\left\{y_{k}\right\}$ contains a subsequence which converges almost everywhere to $a$ Lebesgue measurable function $y$.

In case $\phi(r) \geq \epsilon>0$ for $0<r<+\infty$ for some $\epsilon$ and $p=1$, then this result would follow from [3, Corollary 3.3, p. 418], and in fact a subsequence could be chosen which converged at every point of $I$. An example is given in $\$ 3$ to show that the conclusion of Theorem 1.1 cannot be strengthened to claim the existence of a subsequence converging at every point of $I$ under the given hypotheses of Theorem 1.1 even when $p=1$.

For the reason described above when $p=1$, the main case of interest here is when $\phi(r)$ is not bounded away from zero on $(0,+\infty)$. Since $\phi$ is nondecreasing in $r$ and $\phi(r)$ must be positive for $r$ positive, it is only when $\lim _{r \rightarrow 0} \phi(r)=0$ that $\phi$ is not bounded away from zero. Thus an example of the type of function $\phi$ that we have in mind here would be $\phi(r)=r^{a}$ for $0<$ $\alpha<1$. Note that $\phi(r)=r$ does not satisfy our required restrictions on the function $\phi$, and in fact the sequence $\left\{y_{k}\right\}$, defined by $y_{k}(x)=\sin k x$ for $0 \leq$ $x \leq 1$, will satisfy (1.1) with $M=2, p=1$ and $\phi(r)=r$ but does not have any subsequence which converges almost everywhere on $[0,1]$ [4, Example, p. 162].

In the study of ordinary differential equations, particularly the $n$th order scalar equations, one property of interest is how the solutions behave with respect to crossing each other. In the case of linear equations this amounts to examining the zeros of an arbitrary solution and the sign and magnitude of that solution between zeros. Papers dealing with existence and uniqueness for solutions of $n$-point boundary value problems, disconjugacy or oscillation often involve exactly such considerations. In such papers the solutions are usually at least differentiable so that the difficulty in picking a subsequence that converges in some way (hopefully to something that is in some sense also a solution) from a given sequence of solutions is caused not by the lack of "smoothness" of the functions involved, but rather by the way in which the solutions cross each other.

Given a sequence of solutions of some $n$th order scalar differential equation, one would prefer to extract immediately a subsequence which converged uniformly, along with its derivatives up to order $n$, to a solution $y$ of the same equation and be done with the matter. Unfortunately this is often either not possible at all or else it becomes clear that it is possible only after prolonged examination. A method that is often used instead is to try first to obtain a subsequence converging in some weaker way to something which is then used as the candidate for a solution. The remainder of the analysis is then con- 
cerned with establishing how "smooth" this candidate is and the strongest sense in which it can be considered a solution.

In this regard we might point out one example. For an $n$th order scalar differential equation, if a sequence of solutions $\left\{y_{k}\right\}$ defined on $[c, d]$ is continuous, uniformly bounded in absolute value by $K$, and there is an $N$ so that there are no more than $N$ of the intervals $I_{k j n}$ for each fixed $k, j$, then the hypotheses of our theorem are satisfied using any $\phi$ and $p$ as in the theorem, since

$$
\begin{aligned}
\sum_{n} \phi\left(\mu\left(I_{k j n}\right)\right) & \sup _{x \in I_{k j n}}\left|y_{k}(x)-y_{j}(x)\right|^{p} \\
& \leq \sum_{n} \phi(\mu[c, d]) \sup _{x \in[c, d]}\left|y_{k}(x)-y_{j}(x)\right|^{p} \leq N \phi(d-c)(2 K)^{p} .
\end{aligned}
$$

The restriction on the number of intervals $I_{k j n}$ is a natural one in the study of boundary value or interpolation problems because it is common to assume that if two solutions agree at more than some fixed number of points, then they are identical. In the example described above, a more detailed analysis shows that a subsequence can be extracted which converges at every point of $[c, d][3$, Corollary 3.4, p. 419]. This example does not use the full power of Theorem 1.1, of course, because the theorem allows the possibility that there be infinitely many intervals $I_{k j n}$ for fixed $k$ and $j$ and does not require the functions to be uniformly bounded. This should prove useful in the study of oscillation.

The method of proof used in this paper is similar to that employed by the first author in [3] and [4] and relies heavily on the use of Ramsey's theorem [1, Theorem A] or [2, Theorem A, p. 82].

2. Proof of Theorem 1.1. We may assume that the inequality (1.1) holds for all $k, j$ and that $I$ is an open interval. Let $\left\{J_{i}\right\}$ be an enumeration of all the nonempty open subintervals of $I$ with rational endpoints. It follows from [3, Corollary 2.2, p. 416] applied to $J_{1}$ that either there is a subsequence of $\left\{y_{k}\right\}$, again denoted by $\left\{y_{k}\right\}$, which is monotone on $J_{1}$, or else there is a subsequence of $\left\{y_{k}\right\}$, again denoted by $\left\{y_{k}\right\}$, such that, for $k \neq$ $j, y_{k}(t)>y_{j}(t)$ and $y_{k}(\tau)<y_{j}(\tau)$ for some $t, \tau \in J_{1}$. We now repeat the process described in the previous sentence consecutively on the intervals $J_{2}$, $J_{3}, \cdots$ and then take the diagonal subsequence, denoted by $\left\{y_{k}\right\}$ again. This sequence has the property that on any of the intervals $J_{i}$ it is either monotone on $J_{i}$ for $k$ sufffciently large or else for every $k \neq j$ sufficiently large there are $t, \tau \in J_{i}$ such that $y_{k}(t)>y_{j}(t)$ and $y_{k}(\tau)<y_{j}(\tau)$.

If $\left\{y_{k}\right\}$ so chosen does not converge almost everywhere on $I$ then let 
$E \subset I$ be the set of points where $\left\{y_{k}\right\}$ does not converge. Then $\mu(E)=\epsilon_{0}>$ 0 and $x \in E$ implies $\left\{y_{k}(x)\right\}$ does not converge. We may assume that the subsequence $\left\{y_{k}\right\}$ chosen as described above is not Cauchy in the appropriate $L^{p}$ metric on $E$, for by the completeness of $L^{p}(E)$ and the fact that $L^{p}$ convergence implies convergence in measure, there would be a further subsequence of $\left\{y_{k}\right\}$ which converged almost everywhere on $E$ and we would be done. Thus, there exists an $\epsilon_{1}>0$ and values of $k, j$ arbitrarily large such that $\int_{E}\left|y_{k}-y_{j}\right|^{p} d \mu>\epsilon_{1}$.

Now let $K>M / \epsilon_{1}$ be fixed and choose $R(K)>0$ such that $\phi(r)>K r$ for $0<r<R(K)$. Now choose $a=x_{1}<x_{2}<\cdots<x_{s+1}=b$, where $s \geq 1, a$ is the left endpoint of $I$ and $b$ is the right endpoint of $I$. such that $x_{i}$ $x_{i-1}<R(K) / 16$ for $i=2,3, \cdots, s+1$. For each $i, 2 \leq i \leq s+1$, such that $\left(x_{i-1}, x_{i}\right] \cap E \neq \varnothing$, choose a point $\tau_{i} \in\left(x_{i-1}, x_{i}\right] \cap E$. This will give $q$, $1 \leq q \leq s$, points in $E$ which we rename $t_{1}<t_{2}<\cdots<t_{q}$. Pick mutually disjoint open intervals $U_{1}, U_{2}, \cdots, U_{q}$ from $\left\{J_{i}\right\}$ with $\mu\left(U_{i}\right)<$ $1 / 2 \operatorname{Min}_{2 \leq r \leq s+1}\left|x_{r}-x_{r-1}\right|$ and $t_{i} \in U_{i}$. Now for each $U_{i}$, if $k \neq j$ are sufficiently large depending on $i$, then $y_{k}-y_{j}$ has both signs on $U_{i}$. To see this, suppose not; then for $k \neq j$ sufficiently large, $\left\{y_{k}\right\}$ would be monotone on $U_{i}$, and if $\left|y_{k}\left(t_{i}\right)\right|$ were bounded, then $y_{k}\left(t_{i}\right)$ would converge, contradicting $t_{i} \in E$. On the other hand, if $\left|y_{k}\left(t_{i}\right)\right|$ is unbounded, then choose $k_{0}$ fixed but large enough so that $\left\{y_{k}\right\}$ is a monotone sequence on $U_{i}$ for $k \geq k_{0}$ and such that $y_{k_{0}}\left(t_{i}\right) \neq y_{1}\left(t_{i}\right)$ and such that $y_{k}(t) \geq y_{k_{0}}(t)$ on $U_{i}$ if $y_{k_{0}}\left(t_{i}\right)>y_{1}\left(t_{i}\right)$, or else such that $y_{k}(t) \leq y_{k_{0}}(t)$ on $U_{i}$ if $y_{k_{0}}\left(t_{i}\right)<y_{1}\left(t_{i}\right)$. Then for any value of $k>k_{0}$ sufficiently large we would have

$$
\begin{aligned}
\sum_{n} \phi\left(\mu\left(I_{k 1 n}\right)\right) \sup _{x \in I_{k 1 n}}\left|y_{k}-y_{1}\right|^{p} & \geq \phi\left(\mu\left(I_{k 1 n(k)}\right)\right) \sup _{x \in I_{k 1 n(k)}}\left|y_{k}-y_{1}\right|^{p} \\
& \geq \phi\left(\mu\left(I_{k 1 n(k)} \cap U_{i}\right)\right) \sup _{x \in I_{k 1 n(k)}}\left|y_{k}-y_{1}\right|^{p} \\
& \geq \phi\left(\mu\left(I_{k_{0} \ln ^{1 n}\left(k_{0}\right)} \cap U_{i}\right)\right)\left|y_{k}\left(t_{i}\right)-y_{1}\left(t_{i}\right)\right|^{p}>M,
\end{aligned}
$$

where $n(k)$ is the index such that $t_{i} \in I_{k 1 n(k)}$. This gives a contradiction.

Pick values of $k \neq j$ fixed but sufficiently large so that $y_{k}-y_{j}$ has both signs on each $U_{i}$ for $i=1,2, \cdots, q$. Then for these fixed values of $k$ and $j$ we let $D_{k j}=\left\{x: x \in I,\left|y_{k}(x)-y_{j}(x)\right|>0\right\}=\bigcup_{n} I_{k j n}$. If $I_{k j n}=(c, d)$ for some $n$ then 


$$
\begin{aligned}
I_{k j n} \cap E & =(c, d) \cap E=\left(\bigcup_{i=2}^{s+1}\left((c, d) \cap\left(x_{i-1}, x_{i}\right]\right)\right) \cap E \\
& =\bigcup_{i=2}^{s+1}\left((c, d) \cap\left(x_{i-1}, x_{i}\right] \cap E\right) .
\end{aligned}
$$

The set $(c, d) \cap\left(x_{i-1}, x_{i}\right] \cap E$ is nonempty for at most 4 values of $i$, namely $\alpha, \alpha+1, \beta-1$ and $\beta$ where $c \in\left(x_{\alpha-1}, x_{\alpha}\right]$ and $d \in\left(x_{\beta-1}, x_{\beta}\right]$. Therefore we have

$$
\mu\left(I_{k j n} \cap E\right)=\sum_{i=2}^{s+1} \mu\left((c, d) \cap\left(x_{i-1}, x_{i}\right] \cap E\right) \leq 4(R(K) / 16)<R(K)
$$

for all values of $n$. Thus

$$
\phi\left(\mu\left(I_{k j n}\right)\right) \geq \phi\left(\mu\left(I_{k j n} \cap E\right)\right)>K \mu\left(I_{k j n} \cap E\right)
$$

for all $n$ for which $\mu\left(I_{k j n} \cap E\right)>0$. Thus

$$
\begin{aligned}
& \sum_{n} \phi\left(\mu\left(I_{k j n}\right)\right) \sup _{x \in I_{k j n}}\left|y_{k}-y_{j}\right|^{p} \geq \sum_{n} \phi\left(\mu\left(I_{k j n}\right)\right) \sup _{x \in I_{k j n} \cap E}\left|y_{k}-y_{j}\right|^{p} \\
& \quad \geq K \sum_{n} \mu\left(I_{k j n} \cap E\right) \sup _{x \in I_{k j n} \cap E}\left|y_{k}-y_{j}\right|^{p} \geq K \int_{E}\left|y_{k}-y_{j}\right|^{p} d \mu \geq K \epsilon_{1}>M .
\end{aligned}
$$

This gives a contradiction also, so we conclude that $\mu(E)=0$ and we are done.

3. An example. To show that under the hypotheses of Theorem 1.1 it is not necessarily possible to choose a subsequence which converges at every point of $I$, even if $p=1$, we consider the following example.

For $0 \leq x \leq 1$ and $k=1,2, \cdots$ let

$$
\begin{aligned}
y_{k}(x) & =-4 k^{3} x+k & & \text { for } 0 \leq x \leq 1 / 4 k^{2} \\
& =0 & & \text { for } 1 / 4 k^{2}<x \leq 1 .
\end{aligned}
$$

Choose $\phi(r)=r^{1 / 2}, p=1$ and $M=1$. Then for any $k>j$ we have

$$
\begin{aligned}
\sum_{n} \phi\left(\mu\left(I_{k j n}\right)\right) \sup _{x \in I_{k j n}}\left|y_{k}-y_{j}\right|= & \left(\mu\left(I_{k j 1}\right)\right)^{1 / 2} \sup _{x \in I_{k j 1}}\left|y_{k}-y_{j}\right| \\
& +\left(\mu\left(I_{k j 2}\right)\right)^{1 / 2} \sup _{x \in I_{k j 2}}\left|y_{k}-y_{j}\right| \\
\leq & \left(1 / 4 k^{2}\right)^{1 / 2} k+\left(1 / 4 j^{2}\right)^{1 / 2} j=1 .
\end{aligned}
$$


Thus the hypotheses of Theorem 1.1 are satisfied. No subsequence of $\left\{y_{k}\right\}$ converges at every point of $[0,1]$ because no subsequence of $\left\{y_{k}(0)\right\}$ converges. A similar but more complicated example can be constructed so that there are a countable number of points in $I$ at which each subsequence of $y_{k}$ is monotone increasing and unbounded, and, hence, every subsequence fails to converge on this countable set.

\section{REFERENCES}

1. F. Ramsey, On a problem of formal logic, Proc. London Math. Soc. (2) 30 (1930), 264-286.

2. - The foundations of mathematics, Harcourt-Brace, New York, 1931.

3. K. Schrader, A seneralization of the Helly selection theorem, Bull. Amer. Math. Soc. 78 (1972), 415-419. MR 45 \#8788.

4. - A pointwise convergence theorem for sequences of continuous functions, Trans. Amer. Math. Soc. 159 (1971), 155-163. MR 43 \#6621.

DEPARTMENT OF MATHEMATICS, UNIVERSITY OF MISSOURI, COLUMBIA, MISSOURI 65201 